\title{
Das Korruptionsstrafrecht des StGB
}

\author{
Von Wiss. Mit. Felix Walther, München*
}

Verständnis und Anwendung der Bestechungstatbestände bereiten in Ausbildung wie Praxis nicht selten ganz erhebliche Probleme, die letztlich Folge zu weit geratener Tatbestände sind. Ausgehend von einer begrifflichen Annäherung an das "Korruptionsstrafrecht « sollen im folgenden Beitrag die allen Korruptionstatbeständen zu Grunde liegende Systematik und Struktur aufgezeigt werden, was Voraussetzung für ein teleologisch-restriktives Normverständnis ist. Abschließend werden die internationalen Bezüge des deutschen Strafrechts in diesem Bereich ins Auge gefasst.

»Korruption hat Konjunktur ${ }^{1}$. Schon ein kurzer Blick in die Tagespresse vermag jedem Leser den Eindruck zu vermitteln, er lebe in einer durch und durch korrumpierten Gesellschaft: Nachdem schon dem Siemens-Konzern in der hauseigenen Affäre um »schwarze Kassen « und Schmiergeldzahlungen Strafzahlungen und Gewinnabschöpfungsmaßnahmen in Milliardenhöhe auferlegt worden waren ${ }^{2}$, sah sich zuletzt der DaimlerKonzern nach der Aufdeckung von Bestechungspraktiken zu einer 185 Mio. Euro teuren »Verständigung « mit den US-amerikanischen Behörden veranlasst ${ }^{3}$. Gleichzeitig beschäftigen Affären um »bestechliche« Schiedsrichter und »korrupte«, weil dopende, Profiradfahrer und deren Ärzte die deutsche (Sport-) Öffentlichkeit ${ }^{4}$. Die Liste um medial aufbereitete »Korruptionsskandale « ließe sich beliebig fortsetzten - "gekaufte Betriebsräte bei VW und (wiederum) Siemens (Stichwort AUB), unerlaubte Zahlungen bei MAN, käufliche Doktorväter an Universitäten - diese Stichworte mögen genügen. Der allgemeine Sprachgebrauch geht aber gerne noch weiter: Geht es um vermeintlich voreingenommene Richter, Bonuszahlungen an Banker oder Manager, dubiose Reisebegleiter von Regierungsmitgliedern, die Buchung von Gesprächsterminen mit hochrangigen Politikern oder auch um Datenerhebungs- und Ausspähungsaktionen bei großen deutschen Unternehmen - mit dem »K-Wort« ist man immer schnell zu Stelle ${ }^{5}$.

\section{Korruptionsstrafrecht - eine begriffliche Annäherung}

Die soeben zu Tage getretene, scheinbar beliebige Verwendung des Begriffs der »Korruption" verwundert nur auf den ersten Blick. Anders als es insbesondere dem juristisch interessierten
Betrachter lieb sein mag, handelt es sich hierbei nämlich keineswegs um einen normativ geprägten Begriff. Vielmehr wird der Begriff vom (Straf-)Gesetzgeber weder verwendet noch definiert. Eine gesetzliche Definition würde den Blick darauf verstellen, dass die Korruption ein soziales Phänomen darstellt, welches Beachtung von ganz verschiedenen Wissenschaftszweigen findet. Dementsprechend finden sich sprachwissenschaftliche, theologisch-moralische, soziologische, wirtschafts- und politikwissenschaftliche und auch juristische Definitionsansätze ${ }^{6}$. Zuletzt - und hierbei dürfte es sich um den entscheidenden Punkt handeln - ist die Frage, was der Einzelne als »korrupt « empfindet, eine höchstpersönliche Frage, die von den persönlichen Moral- und Wertvorstellung vorgegeben wird.

Um zu einer auch für rechtspolitisch ambitionierte Strafrechtler handhabbaren Definition zu gelangen bietet sich ein Rückgriff auf die auch vom Bundeskriminalamt herangezogenen Erkenntnisse der kriminologischen Forschung an. Danach ist unter Korruption der »Missbrauch eines öffentlichen Amtes, einer Funktion in der Wirtschaft oder eines politischen Mandats zugunsten eines Anderen, auf dessen Veranlassung oder Eigeninitiative, zur Erlangung eines Vorteils für sich oder einen Dritten, mit Eintritt oder in Erwartung des Eintritts eines Schadens oder Nachteils für die Allgemeinheit (in amtlicher oder politischer Funktion) oder für ein Unternehmen (betreffend Täter als Funktionsträger in der Wirtschaft) $\ll^{7} \mathrm{zu}$ verstehen.

* Der Autor ist wiss. Mit. am Lehrstuhl von Prof. Dr. Helmut Satzger an der Ludwig-Maximilians-Universität München.

1 Wabnitz/Janovsky/Bannenberg Handbuch Wirtschafts- u. Steuerstrafrechts, 3. Aufl, 10. Kap, Rdn. 1.

2 S. nur Süddeutsche Zeitung v. 15.12.2008, S.2; umfassend Graeff/ Schroeder/WolfF Der Korruptionsfall Siemens.

3 Süddeutsche Zeitung v. 3.4.2010, S. 27.

4 Hierzu z. B. Süddeutsche Zeitung v. 1.4.2009, S. 29; instruktiv WeinREICH Korruption im Sport, S. $22 \mathrm{ff}$.

5 S. hierzu auch die Beispiele aus der staatsanwaltschaftlichen Praxis bei Busch StV 2009, 291.

6 Zur Einführung s. DöLling Gutachten C zum 61. Deutschen Juristentag, S. 9 f. ausführlicher v. Arnim/Heiny/Ittner Korruption. Begriff, Bekämpfungs- und Forschungslücken, FÖV Discussion Papers 33 (abrufbar unter http://www.foev-speyer.de/publikationen/pubdb.asp?reihen_id=3).

7 Bundeslagebild Korruption 2008, Kurzfassung, S. 4 (abrufbar unter http://www.bka.de/lageberichte/ko/blkorruption2008.pdf). 


\section{Das Korruptionsstrafrecht des StGB}

Es versteht sich von selbst, dass das Strafrecht nicht in der Lage ist (und wegen seiner Funktion als ultima ratio des Rechtsgüterschutzes auch nicht sein muss), alle unter diese Begriffsbestimmung fallende Verhaltensweisen zu erfassen. Der Begriff des »Korruptionsstrafrechts« kann aber naturgemäß nur an die Bestimmungen des geltenden Rechts anknüpfen. Möglich ist zunächst eine weitere Unterteilung in Korruptionsstrafrecht im engeren und im weiteren Sinne. Zur ersten Gruppe gehören die Strafnormen, die nach ihrem gesetzgeberischen Sinn und Zweck explizit der »Korruptionsbekämpfung $\star^{8}$ dienen und ausschließlich das von der kriminologischen Korruptionsdefinition bezeichnete Verhalten erfassen. Hierunter fallen die Amtsdelikte der $\$ \$ 331-335$ StGB (Vorteilsannahme, Bestechlichkeit, Vorteilsgewährung und Bestechung) und die $\$ \$ 299$, 300 StGB (Bestechlichkeit und Bestechung im geschäftlichen Verkehr) sowie $\$ 108$ b StGB (Wählerbestechung) und $\$ 108$ e StGB (Abgeordnetenbestechung). Das Korruptionsstrafrecht i. w. S. umfasst zusätzlich typische Begleitdelikte der oben genannten Delikte. Zu nennen sind insbesondere ${ }^{9}$ Unterschlagung im Amt oder Dienst ( $\$ 246$ StGB), Geldwäsche ( $\$ 261$ StGB), Betrug ( $\$ 263 \mathrm{StGB})$, Untreue ( $\$ 266$ StGB), Urkundsdelikte ( $\$ \$ 267$ ff. StGB), Submissionsabsprachen ( $\$ 298$ StGB) und Steuerhinterziehung ( $\$ 370 \mathrm{AO})$.

Der folgende systematische Überblick beschränkt sich auf $\$ 299$ StGB sowie $\$ \$ 331 \mathrm{ff}$. StGB, die den Kern des deutschen Korruptionsstrafrechts ausmachen. Dabei soll es weniger darum gehen, eine umfassende Aufbereitung aller Tatbestandsmerkmale und Streitfragen zu liefern. Dies ist - schon aus Platzgründen das Vorrecht der Kommentarliteratur. Vielmehr soll versucht werden, die genannten Korruptionsdelikte in einer geschlossenen Gesamtdarstellung, unabhängig von ihrem gesetzessystematischen Standort gemeinsam darzustellen. Es geht also vor allem darum, das Verständnis der Tatbestände und Problembereiche durch das Herausarbeiten gemeinsamer Tatbestandsmerkmale und Deliktsstrukturen zu erhöhen.

Die übrigen Bestechungstatbestände bleiben auf Grund fehlender Relevanz in Ausbildung und Praxis an dieser Stelle außen vor. Dies gilt insbesondere für die in $\$ 108$ e StGB geregelte Abgeordnetenbestechung. Der Tatbestand wird weithin als lückenhaft und rein symbolisch empfunden. Kritisiert wird insbesondere die Beschränkung auf den Stimmen(ver-)kauf, obwohl die eigentliche Entscheidungsfindung in der folglich korruptionsgefährdeteren Arbeit in den Fraktionen und Ausschüssen stattfände ${ }^{10}$.

\section{Systematik und Rechtsgüter des Korruptionsstraf- rechts}

\section{Die Gesamtsystematik des Korruptionsstrafrechts}

Das Korruptionsstrafrecht i. e. S. beschäftigt sich ausschließlich mit der Bestechung ${ }^{11}$ als Hauptform ${ }^{12}$ der Korruption. "Bestechung " lässt sich typologisch als »regelwidriger Tausch von Vorteilen ${ }^{13}$ charakterisieren. Das Tauschgeschäft besteht zwischen einem Vorteilsgeber und einem Vorteilsnehmer. Das StGB hält nun unterschiedliche Strafnormen bereit, die das Verhalten der beiden "Tauschpartner" kriminalisieren. Wie das folgende Schaubild verdeutlicht ist für die Anwendbarkeit und Abgrenzung der Tatbestände entscheidend, in welchem Lebensbereich sich der Tausch abspielt.

\begin{tabular}{|l|l|l|l|}
\hline \multirow{2}{*}{} & & \multicolumn{2}{|c|}{ Handlung der Person } \\
\cline { 3 - 4 } & & Vorteilsnehmer & Vorteilsgeber \\
\hline $\begin{array}{l}\text { betroffener } \\
\text { Lebensbe- } \\
\text { reich }\end{array}$ & $\begin{array}{l}\text { Öffentliche Ver- } \\
\text { waltung }\end{array}$ & $\$ \S 331,332$ StGB & $\$ \S 333,334$ StGB \\
\cline { 2 - 4 } & $\begin{array}{l}\text { Geschäftlicher } \\
\text { Verkehr }\end{array}$ & $\$ 299$ I StGB & $\$ 299$ II StGB \\
\hline
\end{tabular}

\section{Die Rechtsgüter der Korruptionstatbestände}

Der betroffene Lebensbereich gibt auch die den einzelnen Tatbeständen zu Grunde liegenden Rechtsgüter vor. Die Besonderheiten der verschiedenen Sektoren des öffentlichen Lebens und die hieraus folgende uneinheitliche Beurteilung der durch Bestechungshandlungen verursachten materiellen und immateriellen Schäden sind nach weit überwiegender Auffassung zu unterschiedlich, als dass sich ein einheitliches Schutzgut des gesamten Korruptionsstrafrechts finden ließe. Daher haben sich bislang vereinzelt gebliebene Versuche, ein den $\$ \$ 108 \mathrm{e}, 299,331 \mathrm{ff}$. StGB gemeinsam zu Grunde liegendes Rechtsgut - die "Nichtkäuflichkeit übertragener oder sonst besonders fremdverantwortlicher Entscheidungsmacht sowie das diesbezügliche Vertrauen der Allgemeinheit « ${ }^{14}$ oder auch die »Rechtsinstitute zur Übertragung von Aufgaben $\ll^{15}$ - zu bestimmen, nicht durchgesetzt. Hiervon abgesehen dürfte es aber kaum einen Bereich des Strafrechts geben, in dem derartige Uneinigkeit über die betroffenen Schutzgüter herrscht.

\section{a) Das Rechtsgut der $\$ \$ 331 \mathrm{ff}$. StGB (Korruption in der öffentlichen Verwaltung)}

In der unübersichtlichen Diskussion zum Rechtsgut der $\$ \$ 331 \mathrm{ff}$. StGB werden überwiegend folgende drei Hauptstandpunkte vertreten: Eine verbreitete Ansicht stellt (mit unterschiedlichen Formulierungen) auf die Sachlichkeit staatlicher Entscheidungen bzw. die Lauterkeit des öffentlichen Dienstes $\mathrm{ab}^{16}$. Anderweitig wird das Vertrauen der Allgemeinheit in diese Lauterkeit und Unkäuflichkeit des öffentlichen Dienstes in den Vordergrund gestellt ${ }^{17}$. Die wohl h. M. kombiniert beide Aspekte zu einem »komplexen Rechtsgut«, wonach die Funktionsfähigkeit der öffentlichen Verwaltung und das Vertrauen der Allgemeinheit in die Sachlichkeit und Unabhängigkeit des Verwaltungshandelns gemeinsam geschützt werden ${ }^{18}$.

Eine eingehende Untersuchung der Problematik ${ }^{19}$ kann im Rahmen dieser Abhandlung nicht geleistet werden und ist für den Zweck dieses Beitrags auch nicht zwingend erforderlich.

8 Vgl. zuletzt das »Korruptionsbekämpfungsgesetz« aus dem Jahre 1997 (BGBl. I 2038)

9 Weitere Begleitdelikte bei Greeve/Dörr in: Volk (Hrsg.), Münchener Anwaltshandbuch Verteidigung in Wirtschafts- und Steuerstrafsachen, $\$ 19$ Rdn. $252 \mathrm{ff}$.

10 Zur Kritik Barton NJW 1994, 1098 (1100 f.); LK/Bauer/Gmel 12. Aufl, $\S 108$ e Rdn. 3 f.

11 »Bestechung« ist hier in einem weiteren Sinne zu verstehen, erfasst also auch Vorteilsannahme und -gewährung (zur Abgrenzung von der Bestechung i. e. S. s. unten 3 ).

12 Anders als vielfach angenommen wird, ist "Bestechung « kein Synonym für »Korruption«; z. B. stellt (nach ü. M.) »korruptes« Verhalten wie Nepotismus, Ämterpatronage oder Bereicherung im Amt keine »Bestechung « dar.

13 Grdl. Volk GS Zipf S. 419 (421 ff.); zust. Rönnau in: Achenbach/Ransiek (Hrsg.), Handbuch Wirtschaftsstrafrecht, 2. Aufl, III 2 Rdn. 5; GreEVE/Dörr (o. Fn. 9) \$19 Rdn. 15; SATzGer ZStW 115 (2003), 469 (481).

14 Pragal Die Korruption innerhalb des privaten Sektors und ihre strafrechtliche Kontrolle durch $\$ 299$ StGB, S. 137 ff., 159 f.; DERs. ZIS 2006, 63 ( $71 \mathrm{ff}$.$) .$

15 JAQUES Die Bestechungstatbestände unter besonderer Berücksichtigung des Verhältnisses der $\$ \$ 331 \mathrm{ff}$. StGB zu $\$ 12$ UWG, S. $125 \mathrm{ff}$.

16 Hettinger NJW 1996, 2263 (2268f.); Hölt kemeier Sponsoring als Straftat, S. 85; SSW-StGB/Rosenau $\$ 331$ Rdn. 7; vgl. auch BT-Drs. 7/550 S. 269.

17 So insbes. die Rspr. BGH NJW 2001, 2558 (2559); BGHSt 47, 22 (25); 295 (303); daneben u. a. auch Sch/Sch/Heine 27. Aufl. $\$ 331$ Rdn. 3; ReNGIER BT II, 10. Aufl, $\$ 60$ Rdn. 7; Joecks Studienkommentar StGB, 8. Aufl, Vor $\$ \$ 331 \mathrm{ff}$. Rdn. 1 .

18 BGHSt 30, 46 (48); BGH NJW 1985, 2654 (2656); LACKNER/KÜHL 26. Aufl, $\$ 331$ Rdn. 1; NK/Kuhlen 3. Aufl, $\$ 331$ Rdn. 12; MüKo-StGB/ Korte $\$ 331$ Rdn. 8; BeckOK-StGB/TrüG $\$ 331$ Rdn. 3 .

19 Hierzu instruktiv LK/Sowada 12. Aufl, Vor $\$ 331 \mathrm{Rdn} .29 \mathrm{ff}$; ausführlich Heinrich Der Amtsträgerbegriff im StrafR, S. 209 ff.; Hölt Kemeier (o. Fn. 16) S. $52 \mathrm{ff}$ 
Zweifelhaft ist jedenfalls, inwieweit tatsächlich das Vertrauen als eigenes Rechtsgut geschützt wird ${ }^{20}$. Abgesehen davon, dass es befremdlich erscheint, mit den Mitteln des Strafrechts vermeintliche Allgemeinvorstellungen zu schützen, wäre die strafbefreiende Wirkung einer Genehmigung nach $₫ 331$ III StGB nicht recht erklärlich. In rechtspolitischer Hinsicht bedenklich ist, dass sich mit einem angeblichen Vertrauensverlust die Kriminalisierung auch nur des Anscheins eines Fehlverhaltens von bzw. gegenüber Amtsträgern rechtfertigen lässt.

\section{b) Das Rechtsgut des $\$ 299$ StGB (Korruption im Geschäftsverkehr)}

Nicht weniger verworren stellt sich der Diskussionsstand zum Rechtsgut des $\$ 299$ StGB dar. Weit gehende Einigkeit herrscht noch insofern, dass der Tatbestand jedenfalls und primär das Allgemeininteresse an einem lauteren (auch fairen oder freien) Wettbewerb schütze ${ }^{21}$. Daneben soll er dem Schutz der Mitbewerber, des Geschäftsherrn des bestechlichen Angestellten, dem Verbraucherschutz sowie dem Schutz der Allgemeinheit vor Verteuerung und Verfälschung marktgerechter Preise die$n^{22}$. Über das innere Verhältnis und die Prioritätenfolge innerhalb dieses »Rechtsgutsgemenges« herrscht wenig Klarheit. In der Tat liegt hier der Verdacht nahe, dass jeder nebensächliche Schutzreflex sogleich zum Rechtsgut avanciert, und somit der nach h. M. vorrangige Aspekt - Sicherung fairer Wettbewerbsbedingungen - verdunkelt wird ${ }^{23}$.

Der »lautere Wettbewerb« als Hauptaspekt der Rechtsgutsdefinition der h. M. lässt sich freilich einer weiteren Präzisierung zuführen. Es empfiehlt sich hierzu, nochmals auf das Wesen der Bestechung - regelwidriger Tausch von Vorteilen - zurück zu greifen. Die Regelwidrigkeit bei der Bestechung im geschäftlichen Verkehr ergibt sich nun daraus, dass in Folge der Korruption das Leistungsprinzip als Maßstab für wettbewerbsrelevante Entscheidung ersetzt wird und folglich die Mechanismen des marktwirtschaftlichen Leistungswettbewerbs ausgehebelt wer$\operatorname{den}^{24}$. Mit anderen Worten: Am Markt setzt sich nicht der Anbieter des leistungsstärksten Produkts durch, sondern der des höchsten Schmiergeldes.

\section{Die Systematik der einzelnen Korruptionstatbestände}

Auch die den einzelnen Lebensbereichen zugeordneten Strafvorschriften weisen nochmals eine jeweils eigene Binnensystematik auf.

Die auf den ersten Blick verwirrend anmutende Systematik der Tatbestände zur Amtsträgerkorruption ( $\$ \$ 331 \mathrm{ff}$. StGB) erschließt sich, wenn man folgende Punkte im Auge behält. Zunächst ist die spiegelbildliche Ausgestaltung der Tatbestände für die (Vorteils-)Geber und Nehmerseite zu beachten. Einer Vorteilsannahme des Nehmers ( $\$ 331 \mathrm{StGB})$ korrespondiert in aller Regel $^{25}$ eine Vorteilsgewährung des Gebers $(\$ 333$ StGB) des Gebers, während die Bestechlichkeit $(\$ 332 \mathrm{StGB}$, sog. passive Bestechung) der Bestechung ( $\$ 334 \mathrm{StGB}$, sog. aktive Bestechung) entspricht. Die Unterscheidung zwischen Vorteilsannahme bzw. -gewährung einerseits und Bestechlichkeit bzw. Bestechung andererseits richtet sich danach, ob der Vorteil für die (pflichtgemäße) Dienstausübung (dann $\$ \$ 331,333 \mathrm{StGB}$ ) oder für eine pflichtwidrige Diensthandlung (dann $\$ \$ 332,334$ StGB) geleistet wird. Die Strafzumessungsvorschrift des $\$ 335$ StGB normiert daneben erhöhte Strafdrohungen für besonders schwere Fälle $\operatorname{der} \S 332$ StGB und $\$ 334$ StGB.

\begin{tabular}{|l|l|l|l|}
\hline & & Vorteilsnehmer & Vorteilsgeber \\
\hline $\begin{array}{l}\text { Verhalten } \\
\text { des Neh- } \\
\text { mers }\end{array}$ & Dienstausübung & $\S 331$ StGB & $\$ 333$ StGB \\
\cline { 2 - 4 } & $\begin{array}{l}\text { Pflichtwidrige } \\
\text { Diensthandlung }\end{array}$ & $\S 332$ StGB & $\S 334$ StGB \\
\hline
\end{tabular}

Für die Fallbearbeitung ist daneben von Bedeutung, dass nach h. M. ${ }^{26} \$ \$ 332,334$ StGB Qualifikationen zu den Grundtat- bestände $\$ \$ 331,332$ StGB darstellen, mit deren Prüfung folglich $\mathrm{zu}$ beginnen ist. Schließlich ist die korruptionsstrafrechtliche Bewertung des Verhaltens von Geber und Nehmer abschließend in $\$ \$ 331,332$ StGB bzw. $\$ \$ 333,334$ StGB geregelt, so dass der Nehmer nicht etwa noch zusätzlich als Teilnehmer an der Tat des Gebers zu bestrafen ist.

Bestechlichkeit und Bestechung im geschäftlichen Verkehr werden für Geber und Nehmer gesetzessystematisch in einer Vorschrift, $\$ 299$ StGB, geregelt. $\$ 300$ StGB sieht einen erhöhten Strafrahmen für besonders schwere Fälle vor, während $\$ 301$ StGB die Verhängung des erweiterten Verfalls ( $\$ 73 \mathrm{~d}$ StGB) erlaubt ${ }^{27}$. Von praktischer Bedeutung ist, dass gemäß $\$ 300$ StGB Taten nach $\$ 299$ StGB nunmehr ${ }^{28}$ auch von Amts wegen verfolgt werden können.

\section{Problemkreise der Korruptionsdelikte}

\section{Täterkreis}

Als Vorteilsgeber kommt grds. Jedermann in Betracht. Die $\$ \$ 299$ II, 333, 334 StGB stellen sog. Allgemeindelikte dar. Die für die Nehmerseite konzipierten Tatbestände ( $\$ \$ 299$ I, 331, 332 StGB) sind als Sonderdelikte ausgestaltet und können folglich nur von einem bestimmten Personenkreis täterschaftlich ${ }^{29}$ begangen werden. Eine genaue Umschreibung der in Betracht kommenden Vorteilsnehmer ist aber auch für die strafrechtliche Bewertung der Geberseite von essentieller Bedeutung, da der Vorteilsnehmer aus dem fraglichen Personenkreis stammen muss, um im strafrechtlichen Sinne (aktiv) »bestochen« werden zu können.

\section{a) Täter der $\$ \$ 331,332 S t G B$}

Vorteilsannahme ( $\$ 331$ StGB) und Bestechlichkeit ( $\$ 332$ StGB) zählen zu den sog. Amtsdelikten, die nur von "Amtsträgern« oder »für den öffentlichen Dienst besonders Verpflichteten« begangen werden können. Die fraglichen Begriffe sind in $\$ 11 \mathrm{I}$ Nrn. 2-4 StGB legaldefiniert. Diese komplizierte Regelung vermag freilich insbesondere dann, wenn öffentliche Aufgaben mit Hilfe Privater (Stichwort: public private partnerships) oder in privatrechtlicher Organisationsform wahrgenommen werden, nur begrenzt weiterzuhelfen. Die im Einzelfall schwierigen Abgrenzungsfragen sollen im Rahmen dieses Beitrages nicht weiter geklärt werden ${ }^{30}$. Vor der Annahme einer Amtsträgerschaft i. S. d. $\$ \$ 331-334$ StGB sollte man sich jedoch grds. die »Testfrage« stellen, ob durch die fragliche Handlung tatsächlich die

20 Zur Kritik Dölling (o. Fn. 6) S. 49; Höltkemeier (o. Fn. 16) S. 58 f.; LK/SOWADA Vor $\$ 331 \mathrm{Rdn} .35 \mathrm{ff}$.

21 BGH NJW 2006, 3290 (3298); SSW-StGB/Rosenau \$299 Rdn. 1; vgl. auch BT-Drs. 16/6558 S. 13.

$22 \mathrm{Zu}$ diesen (Neben-)Interessen LK/Tiedemann 12. Aufl, $\$ 299$ Rdn. 2 ff.; NK/DANNECKER $\$ 299$ Rdn. 5 ff.

23 Rönnau (o. Fn. 13) III 2 Rdn. 6 m. w. N.

24 Ausführlich und überzeugend Koepsel Bestechlichkeit und Bestechung im geschäftlichen Verkehr ( $\$ 299$ StGB), S. 99 ff.; zust. RönnaU (o. Fn. 13) III 2 Rdn. 6 (Fn. 37).

25 Ausnahmen können sich ergeben, wenn nur ein Beteiligter Vorsatz bzgl. einer pflichtwidrigen Diensthandlung hat oder ein einseitiges Fordern bzw. Anbieten eines Vorteils fehlschlägt.

26 S. nur Rengier (o. Fn. 17) \$60 Rdn. 3; anders die Staatswillensverfälschungslehre (s. oben 2, a), die $\$ \$ 332,334$ StGB als Grunddelikte und $\$ \$ 331,333$ StGB als abstrakte Gefährdungsdelikte begreift (s. HöLTKEMEIER [o. Fn. 16] S. 56); zum Ganzen ausf. RÜDIGER Schutzinteresse und Deliktsstruktur der »Bestechungsdelikte« ( $\$ \$ 331 \mathrm{ff}$. StGB), S. $65 \mathrm{ff}$.

27 Der in $\$ 300$ II StGB genannte $\$ 43$ a StGB ist durch Urteil des BVerfG vom 20.3. 2002 (BVerfGE 105, 135) mit Gesetzeskraft für nichtig erklärt worden (BGBl. I 1340).

28 Die Vorschrift wurde erst 1997 eingefügt. Zuvor war die Tat ausschließlich auf Antrag zu verfolgen.

29 Ein Außenstehender (sog. extraneus) kann sich hingegen nur als Teilnehmer strafbar machen, dessen Strafe gem. $\$ 28$ I StGB zu mildern ist.

30 Dazu ausführlich F. WALther JURA 2009, 421. 
Rechtsgüter der »Lauterkeit der öffentlichen Verwaltung bzw. das diesbezügliche Vertrauen der Allgemeinheit« berührt werden.

\section{b) Täter des $\$ 299$ I StGB}

Fall 1: A betreibt als Alleinunternehmer einen Friseursalon. Als die Erneuerung der Waschbecken ansteht, machen die Firmen $X$ und $Y$ Angebote. Bei Firma X kosten die neuen Becken und deren Einbau am wenigsten. Firma Y bietet dem A aber an, ihm einen exklusiven Spiegel für sein privates Badezimmer als "Zugabe zu liefern.

Variante: Um eine persönliche Haftung für geschäftliche Verbindlichkeiten zu vermeiden, gründet A eine GmbH und tritt als Alleingesellschafter und -geschäftsführer auf.

Das Gesetz nennt als Täter der Bestechlichkeit im geschäftlichen Verkehr in $\$ 299$ I StGB »Angestellte oder Beauftragte eines geschäftlichen Betriebes«. Unter Berufung auf das Rechtsgut des lauteren Wettbewerbs wird überwiegend eine weite Auslegung der beiden Begriffe gefordert ${ }^{31}$. Angestellter ist, wer vertraglich oder faktisch in einem Dienstverhältnis zum Inhaber eines Geschäftsbetriebs steht und dessen Weisungen unterworfen is ${ }^{32}$. Dem Begriff des Beauftragten kommt demgegenüber eine Auffangfunktion $\mathrm{zu}$, so dass hierunter alle Personen zu verstehen sind, die - ohne Inhaber oder Angestellter eines Betriebes zu sein - auf Grund ihrer Stellung im Betrieb berechtigt und verpflichtet sind, für den Betrieb geschäftlich zu handeln, und unmittelbar oder mittelbar Einfluss auf die im Rahmen des Geschäftsbetriebes zu treffenden Entscheidungen ausüben können ${ }^{33}$. Der Begriff des geschäftlichen Betriebs umfasst jede auf gewisse Dauer betriebene Tätigkeit im Wirtschaftsleben, die den Austausch von Leistungen und Gegenleistungen beinhaltet ${ }^{34}$. Privathaushalte, die nur sporadisch am Markt tätig sind, sind demnach kein "geschäftlicher Betrieb«, so dass deren Beauftragte (z. B. Architekten) nicht i. S. d. \$299 I StGB bestochen werden können.

So klar der Täterkreis damit umschrieben scheint, so unklar ist häufig die Abgrenzung im Einzelfall. Die Unklarheiten resultieren in erster Linie daraus, dass nach dem eindeutigen Gesetzeswortlaut der Geschäftsinhaber selbst als Täter des $\$ 299$ I StGB ausscheidet, was vielfach als rechtspolitisch verfehlt angesehen wird ${ }^{35}$. In einigen Fällen - z. B. Vorstände von Aktiengesellschaften, GmbH-Geschäftsführern - lassen sich bei formeller Betrachtung die Unternehmensleiter aber durchaus als »Beauftragte « des geschäftlichen Betriebs begreifen ${ }^{36}$. Dass dieses Verständnis in letzter Konsequenz nicht durchgehalten werden kann, soll Fall 1 zeigen. Hier erschiene es kaum überzeugend, die Tätereigenschaft des A im Ausgangsfall und in der Variante verschieden zu beurteilen. In beiden Fällen ist A faktisch Unternehmensinhaber und kommt deswegen als Täter des $\$ 299$ I StGB nicht in Betracht ${ }^{37}$.

\section{Das Bestechungsmittel: Vorteil}

Fall 2: Staatsanwalt S ermittelt wegen Schwarzfahrens ( $\$ 265$ a I Var. 3 StGB) gegen Zeitungsredakteur Z. Da Z sich bisher noch nichts $z$ U Schulden hat kommen lassen, stellt $S$ das Verfahren gemäß $\$ 153$ a StPO gegen Zahlung von $500 €$ an eine gemeinnützige Hilfsorganisation ein.

Variante: $Z$ hatte $S$ zu verstehen gegeben, er werde ihn in einem künftigen Artikel als »Kämpfer für Recht und Ordnung «arstellen.

Fall 3: Um die Lieferung fünf neuer Kopierer für das Unternehmen $S$ bewerben sich X und Y. Der für die Vergabe zuständige Angestellte A gibt $X$ zu verstehen, dass er den Auftrag erhalte, wenn er statt der angebotenen zweijährigen Garantie einen fünfjährigen Vorort-Service anbiete.

Fall 4: Ausgangssituation wie Fall 3. Allerdings hat X erfahren, dass A Unternehmensgelder veruntreut hat. Er stellt A in Aussicht, dessen Vorgesetzten zu informieren, wenn er nicht den Auftrag erhalte.
Im Mittelpunkt aller Tatbestände des Korruptionsstrafrechts steht der »Vorteil« als Mittel der Bestechung. Dieser »Vorteil« stellt die Bestechungsleistung dar, die vom Täter der Bestechung an den Täter der Bestechlichkeit geleistet wird bzw. werden soll. Nach der von der vom BGH in ständiger Rechtsprechung gebrauchten Formel ist ein Vorteil jede Leistung, auf die der Leistungsempfänger keinen Rechtsanspruch hat und die seine wirtschaftliche, rechtliche oder auch nur persönliche Lage unmittelbar oder mittelbar objektiv verbessert ${ }^{38}$.

\section{a) materielle und immaterielle Vorteile}

Vorteile i. S. d. $\$ \$ 108$ b/e, 299, 331 ff. StGB sind zunächst alle Zuwendungen, die eine wirtschaftliche Besserstellungen des Vorteilsempfängers bewirken. Zu nennen sind hier das klassische Bargeld, Einladungen zum Essen oder kulturellen oder sportlichen Veranstaltungen, die Einräumung von Rabatten, die Gewährung von Darlehen $\mathrm{usw}^{39}$. Zu beachten ist in diesem Zusammenhang lediglich, dass durch das Abstellen auf die "wirtschaftliche« Besserstellung der Vorteilsbegriff eine gewisse Objektivierung erfährt und dass die Vorteilsgewährung sich nicht im Sinne einer "Stoffgleichheit « vermögensmindernd beim Geber auswirken muss.

Daneben erfasst das Gesetz auch sog. immaterielle Vorteile. Hierunter sollen Ehrenämter, Ehrungen, sexuelle Zuwendungen, Karrierechancen usw. fallen ${ }^{40}$. In der Variante von Fall 2 könnte daher grds. der »Gefälligkeitsartikel« einen immateriellen Vorteil darstellen. Literatur und neuere Rechtsprechung haben aber erkannt, dass sich letztlich fast jede Erregung positiver Gefühle als »Vorteil« verstehen ließe und wollen deshalb nur solche »immateriellen Vorteile« anerkennen, die objektiv messbar und daher dem Nachweis zugänglich sind ${ }^{41}$.

$\mathrm{Ob}$ hiermit wirklich eine allgemein gültige Formel gefunden ist, bleibt aber zweifelhaft. Denn wie ein immaterieller Vorteil objektiv (!) messbar sein soll, ist nicht ersichtlich. Soweit es hingegen um vermeintlich »eindeutige« Fälle geht (z. B. Gewährung von Geschlechtsverkehr, Zuerkennung von Exspektanzen), sind diese regelmäßig kommerzialisierbar und daher als materielle Vorteile einzuordnen. Bzgl. des Gefälligkeitsartikels in der Variante von Fall 2 wäre nach den großzügigen Maßstäben der h. M. eine relevante Besserstellung des S wohl zu bejahen ${ }^{42}$.

\section{b) Eigen- und Drittvorteile}

Dass immaterielle Vorteile überhaupt unter den Begriff des »Vorteils« fallen sollen, wird erst dann verständlich, wenn man sich vor Augen führt, dass die $\$ \$ 331 \mathrm{ff}$. StGB a. F. und $\$ 12$ UWG a. $\mathrm{F}^{43}$ bis 1997 nur solche Vorteile erfassten, die dem Amtsträger bzw. dem Angestellte selbst zukommen sollten. Um eine Umgehung der Bestechungsverbote durch die Zwischenschaltung z. B.

31 RGSt 66, 119 (120); 72, 62 (63f.); SSW-StGB/RosenAu \$299 Rdn. 7, 9; MüKo-StGB/Diemer/Krick \$299 Rdn. 4 m. w. N.

32 Fischer 57. Aufl, \$299 Rdn. 9; SSW-StGB/Rosenau \$299 Rdn. 7.

33 RGSt 68, 70 (74 f.); 263 (270); BGHSt 2, 396 (401); BGH NJW 1968, 1572 (1573).

34 FisCher $\$ 299$ Rdn. 4; LACKNER/KÜHL $\$ 299$ Rdn. 2.

35 S. nur Bürger wistra 2003, 130 (132); Höltkemeier (o. Fn. 16) S. $169 \mathrm{ff}$.

36 So auch die h. M.; vgl. RGSt 66, 81; BGHSt 20, 210.

37 So auch Sch/Sch/Heine $\$ 299$ Rdn. 7; LK/Tiedemann \$299 Rdn. 14; ausf. Hanft Strafrechtliche Probleme der Einmann-GmbH, S. 159 ff.; beachtliche Gründe gegen die Täterqualität des AG-Vorstandes bei Brand/Wostry WRP 2008, 637 (639).

38 BGHSt 35, 128 (133); 47, 295 (304); BGH NStZ 2008, 216 (217).

39 Weitere Beispiele und Nachweise bei Fischer $\$ 331$ Rdn. 11 b; Sch/Sch/ HeIne $\$ 331$ Rdn. 18.

40 Beispiele bei MüKo-StGB/Konte $\$ 331$ Rdn. 71; BeckOK/Trüg $\$ 331$ Rdn. 18.1.

41 BGHSt 47, 295 (305); BGH NJW 2003, 763 (766); SATZger ZStW 115 (2003), 469, 478; Rengier (o. Fn. 17) \$60 Rdn. 10

$42 \mathrm{Vgl}$. NK/Kuhlen $\$ 331 \mathrm{Rdn}$. 40; Fischer $\$ 331 \mathrm{Rdn} .11 \mathrm{c}$. 43 Als Vorgängernorm von $\$ 299$ StGB. 
naher Angehöriger als Vorteilsempfänger zu verhindern, deutete die ältere Rechtsprechung solche materiellen Drittzuwendungen häufig in immaterielle Eigenvorteile $\mathrm{um}^{44}$. Diese Vorgehensweise ist durch die eindeutige Einbeziehung Dritter als Vorteilsempfänger überflüssig geworden. Andererseits hat die Erstreckung auf Drittvorteile dazu geführt, dass eine ganze Reihe sozial unverfänglicher oder sogar erwünschter Verhaltensweisen dem Wortlaut nach bestraft werden müsste ${ }^{45}$.

So hat in Fall 2 der Amtsträger S für eine Diensthandlung (Einstellung des Verfahrens) einen materiellen Vorteil (500€) für einen Dritten (die Hilfsorganisation) gefordert bzw. sich versprechen lassen. Dass es seltsam anmuten würde, hier erst über einen (übergesetzlichen) Rechtfertigungsgrund zur Straflosigkeit zu gelangen ${ }^{46}$, muss nicht näher begründet werden. In Fall 3 scheint $\$ 299$ I StGB ebenfalls erfüllt zu sein: Der Angestellte A fordert einen Vorteil (Vorort-Service) für einen Dritten (das Unternehmen) als Gegenleistung für eine Bevorzugung im Wettbewerb. Auch hier wäre eine Bestrafung des A aber offensichtlich unangebracht. Richtigerweise ist die Lösung für derartige Fälle über das normative Merkmal der Unrechtsvereinbarung zu suchen (dazu unten $4, \mathrm{~d}, \mathrm{bb}$ ).

\section{c) Ausbleiben eines Übels als Vorteil}

In Fall 4 stellt sich die Frage, ob in der Beibehaltung des status quo ein »Vorteil« i. S. d. Bestechungsdelikte liegen kann. Der BGH ${ }^{47}$ hat in der Tat unter Hinweis auf die Rechtsprechung des Reichsgerichts ${ }^{48}$ in der Nichtveröffentlichung dienstlicher Verfehlungen einen »Vorteil« gesehen und ist dafür im Schrifttum stark kritisiert worden ${ }^{49}$. Den Weg zur Lösung weist das Parallelproblem der »Drohung mit Unterlassen « bei $\$ 240$ StGB. Dort ist zutreffenderweise eine "Drohung mit einem empfindlichen Übel dann zu verneinen, wenn dem Opfer lediglich die Beibehaltung des status quo unter Ausbleiben einer Verbesserung in Aussicht gestellt wird ${ }^{50}$. Übertragen auf den Vorteilsbegriff bedeutet dies, dass ein »Vorteil« nur dann anzunehmen ist, wenn dem Empfänger angeboten wird, eine ohnehin anstehende negative Entwicklung abzuwenden, und somit dessen Handlungsspielraum erweitert wird. Geht es hingegen nicht nur um die Beibehaltung des status quo, sondern um die Herbeiführung eines Übels (und dessen anschließende Abwendung), so ist darin schon dem Wortsinn nach keine »Besserstellung « $\mathrm{zu}_{\text {sehen }}{ }^{51}$. Die entgegengesetzte Lösung würde zu dem befremdlichen und wertungswidersprüchlichen Ergebnis führen, dass derjenige, der einen anderen mit der Androhung eines empfindlichen Übels nötigt (!) diesem gleichzeitig einen »Vorteil « anbietet.

Für Fall 4 bedeutet dies, dass das Angebot an A nur dann einen »Vorteil« darstellt, wenn dessen Machenschaften ohne Zutun des $\mathrm{X}$ ohnehin bald bekannt geworden wären. Hätte dagegen erst X die Verfehlungen publik gemacht, so kann im Ausbleiben dieses Übel kein »Vorteil« liegen. Vielmehr wäre X nach $\$ 240$ StGB zu bestrafen.

\section{d) Sozialadäquanz und Geringwertigkeit}

Fall 5: Aus Dankbarkeit für eine schnelle Auskunft möchte Anwältin A den Bediensteten im Grundbuchamt »eine Kleinigkeit für die Kaffeekasse « überreichen.

Variante: A bemüht sich um ein attraktives Mandat der Firma $X$. Sie lädt deren Prokuristen $P$ zu einem »Geschäftsessen « in ein Lokal der gehobenen Klasse ein.

Man ist sich im Ergebnis weitgehend einig, dass die Annahme bzw. Gewährung geringwertiger oder sozial anerkannter Zuwendungen nicht von den Korruptionstatbeständen erfasst werden sollen. Der dogmatische Weg zu dieser Lösung ist allerdings steinig. So wird vielfach vorgeschlagen, geringwertige oder sozialadäquate Zuwendungen aus dem Begriff des »Vorteils« auszunehmen $^{52}$. Dies vermag nicht zu überzeugen: Da die einge- schränkte Vorteilsdefinition dann für alle Tatbestände gelten müsste, hätte dies die inakzeptable Folge, dass der Kauf pflichtwidriger Diensthandlungen mangels "Vorteils« nicht gem. $\$ \$ 332,334$ StGB bestraft werden könnte. In Fall 5 (einschließlich der Variante) hat A daher jeweils einen »Vorteil« i.S.d. $\$ \$ 299$ II, 333 StGB angeboten. Allerdings bleibt in diesen Fällen die Regelwidrigkeit des Austauschverhältnisses besonders genau zu prüfen (s. dazu unten $4, \mathrm{~d}$ ).

\section{Tathandlung}

Eine Erläuterung der Tathandlungen kann gemeinsam für $\$ \$ 331 \mathrm{ff}$. und $\$ 299$ StGB erfolgen, da dieselben Verhaltensweisen genannt werden. Der Nehmer muss einen Vorteil fordern, sich versprechen lassen oder annehmen ( $\$ \$ 331 \mathrm{f} ., 299$ I StGB). Der Nehmer bietet den Vorteil an, verspricht oder gewährt ihn ( $\$ \$ 333$ f., 299 II StGB). Entgegen eines ersten Anscheins handelt es sich hierbei keineswegs um sich überschneidende oder teils redundante Begriffe. Das Verständnis für den Bedeutungsgehalt der einzelnen Handlungsalternativen erhöht sich durch eine »Pärchenbildung «, d. h. die Herstellung eines stufenweisen Entsprechungsverhältnisses zwischen den Verhaltensweisen des Nehmers und Gebers.

Auf der ersten »Verhandlungsstufe « fordert der Nehmer einen Vorteil durch einseitiges ausdrücklich oder schlüssig erklärtes Verlangen zur Kenntnis des Gebers oder eines Mittelsmannes ${ }^{53}$. Wird aber der Geber zuerst tätig, so handelt es sich um ein Anbieten. Hierunter zu verstehen ist die auf Abschluss einer Unrechtsvereinbarung gerichtete ausdrückliche oder stillschweigende Erklärung zur Kenntnis des Nehmers oder eines für diesen tätigen Dritten ${ }^{54}$. Reagiert der Nehmer zustimmend auf ein einseitiges Angebot des Gebers, so verwirklicht er die Handlungsalternative des Sich-versprechen-lassens. Entsprechend verspricht der Geber einen Vorteil dann, wenn er auf ein Fordern des Nehmers eingeht. Um den vollendeten Leistungsaustausch handelt es sich schließlich beim Annehmen, wenn der Nehmer den angebotenen Vorteil tatsächlich empfängt ${ }^{55}$. In diesem Fall hat gleichzeitig der Geber den Vorteil gewährt.

\section{Unrechtsvereinbarung und Gegenleistung des Bestechungsadressaten}

Fall $6^{56}$ : U war Vorstandsvorsitzender des Energiekonzerns EnBW, der als Hauptsponsor der Fußball-WM 2006 auftrat. Im Rahmen eines umfassenden Sponsoringskonzepts versandte er Weihnachtsgrußkarten mit beigelegten Gutscheinen für Logenplätze bei einem WM-Spiel u.a. an Landesminister und einen Staatssekretär im Bundesministerium für Umwelt.

Fall 7 a) Der Bauunternehmer U verwirklicht in der Stadt S immer wieder Bauvorhaben und steht deswegen in engem Kontakt mit

44 Vgl. nur RGSt 77, 75 (78); BGHSt 14, 123 (128); OLG Karlsruhe NStZ 2001, 654 (655).

$45 \mathrm{Zu}$ den Hauptstreitfällen Parteispenden, Drittmittelwerbung und Sponsoring vgl. instruktiv BAnnenberg (o. Fn. 1) 10. Kap. Rdn. 72 ff.; LK/ SOWADA $\$ 331 \mathrm{Rdn} .76 \mathrm{ff}$.

46 Dazu P. Cramer FS Roxin S. 945 (950); St. Cramer wistra 1999, 414. 47 BGH NStZ 1985, 497 m. abl. Anm. Marcelli.

48 RGSt 64, 374 (375f.), hier wurde aber wohl die Zusage, ein Mietverhältnis fortsetzen zu dürfen, als Vorteil verstanden und nicht etwa die gleichzeitige Drohung, es zu beenden.

49 Bernsmann/Gatzweiler Verteidigung bei Korruptionsfällen, Rdn. 205 ff.; WAGNER JZ 1987, 594 (603 f.).

50 Vgl. Roxin JR 1983, 333 (336).

51 Vgl. auch NK/KuHLen $\$ 331$ Rdn. 36 f.; LK/Sowada $\$ 331$ Rdn. 35. 52 So BGHSt 33, 336 (339); SK/Rudolphi/STEIN $\$ 331$ Rdn. 19 und 23. 53 BGHSt 10, 237 (241); 15, 239 (242); 47, 22 (29).

$54 \mathrm{Sch} / \mathrm{Sch} / \mathrm{Heine} \$ 333 \mathrm{Rdn} .4$; MüKo-StGB/Korte $\$ 333 \mathrm{Rdn} .10$. 55 BGHSt 39, 45 (46); BGH NJW 1987, $1340 \mathrm{f}$

56 In Anlehnung in BGH NJW 2008, $3580=$ SAtzger JK 5/09, StGB $\$ 333 / 2$. 
dem städtischen Baureferat. Als der neue Referatsleiter $M$ seinen Dienst antritt, lädt U ihn mit dem Wunsch »auf gute Zusammenarbeit« zu einem Golfturnier ein.

b) Wenig später hat das Baureferat einen Auftrag zu vergeben. Es gibt mehrere Bewerber. Die Vergabeentscheidung steht im Ermessen des M. Seine Ehefrau erhält kurz darauf von U ein schickes Kleid, dessen Wert $M » d o c h$ bitte bei der Vergabe berücksichtigen möge«.

Variante: $M$ ist leitender Angestellter der Firma F., die eine neue Produktionshalle bauen will.

\section{a) Einleitendes zur sog. "Unrechtsvereinbarung "}

Aus dem Wesen der Korruption - Tausch von Vorteilen - folgt, dass neben die bloße Vorteilsannahme auch eine selbstständige "Gegenleistung " des Nehmers treten muss ${ }^{57}$. Im Gesetzestext kommt dieses Gegenseitigkeits- oder Äquivalenzverhältnis dadurch zum Ausdruck, dass der Vorteil »für« (so in $\$ \$ 331,333$ StGB) oder »als Gegenleistung [für] « (so in $\$ \$ 299,332,334$ $\mathrm{StGB}$ ) ein bestimmtes Verhalten des Nehmers geleistet werden muss. Für die Rechtspraxis bedeutet dies, dass der jeweils isolierte Nachweis einer Vorteilsgewährung und einer Gegenleistung noch nicht ausreicht. Vielmehr ist festzustellen, dass die Zuwendung nach dem ausdrücklichen oder stillschweigenden Einverständnis der Beteiligten ihren Grund gerade in der Gegenleistung des Nehmers hat ${ }^{58}$. Dies bedeutet: Sobald nicht auszuschließen ist, dass eine Zuwendung lediglich aus Höflichkeit, den Regeln der allgemeinen Verkehrssitte oder anderen Beweggründen erfolgt, die nicht in Zusammenhang mit der tatbestandlich umschriebenen Gegenleistung des Nehmers stehen, scheidet die Bestrafung aus einem Korruptionsdelikt aus. In Fall 6 hatte das Tatgericht - für den BGH als Revisionsinstanz insoweit bindend - nicht ausschließen können, dass die Versendung der WM-Tickets allein zu Werbezwecken genutzt werden sollte, um die Veranstaltung aufzuwerten und die Rolle der EnBW als Sponsor der Veranstaltung hervorzuheben ${ }^{59}$. Da solche Werbezwecke keine taugliche Gegenleistung (s. dazu sogleich) darstellen, war der Angeklagte freizusprechen.

Die überragende Bedeutung dieser (angestrebten) ${ }^{60}$ Unrechtsvereinbarung rechtfertigt ihre Bezeichnung als das »Kernstück aller Bestechungsdelikte « ${ }^{61}$. Für ein korrektes Verständnis in Fallbearbeitung und Praxis sind insbesondere folgende Punkte von Bedeutung:

- Die Gegenleistung des Nehmers muss in keinem Fall tatsächlich vorgenommen werden. Vielmehr ist es ausreichend, dass die Parteien sie zum Gegenstand ihrer Unrechtsvereinbarung gemacht haben.

- Das zeitliche Verhältnis zwischen Vorteilszuwendung und Gegenleistung wird in den einzelnen Tatbeständen unterschiedlich geregelt: Während bei $\$ \$ 331 \mathrm{ff}$. StGB der Vorteil in Hinblick auf eine vergangene oder zukünftige Verhaltensweise des Nehmers geleistet wird ${ }^{62}$, ist bei $\$ 299$ StGB nur die Zuwendung für eine zukünftige Bevorzugung im Wettbewerb erfasst.

- Da die Unrechtsvereinbarung ein starkes subjektives Element aufweist, ist ihre Einordnung im Prüfungsaufbau zweifelhaft. Teilweise wird sie als subjektives Tatbestandsmerkmal, teils als eigener Prüfungspunkt zwischen objektivem und subjektivem Tatbestand verortet. Da sich die (angestrebte) Vereinbarung aber auch in der Tathandlung objektiv manifestieren muss, spricht nichts dagegen, sie im objektiven Tatbestand anzusprechen.

\section{b) Gegenleistungen bei $\$ \$ 331 \mathrm{ff}$. StGB: Dienstausübung/} Diensthandlung/Dienstpflichtverletzung

Der entscheidende Unterschied zwischen bloßer Vorteilsannahme bzw. -gewährung einerseits und der Bestechlichkeit und Bestechung andererseits ist, dass bei den $\$ \$ 331,333$ StGB die Vorteilsgewährung für die bloße Dienstausübung ausreichend ist, während $\$ \$ 332,334$ StGB auf eine pflichtwidrige Diensthandlung abstellen. Besonderheiten gelten für richterliche Tätigkeiten. Erfolgt die Zuwendung um einer pflichtgemäßen richterlichen Handlung ${ }^{63}$ willen, sind $\$ \$ 331$ II, 333 II StGB einschlägig. Steht der "Kauf « einer pflichtwidrigen richterlichen Handlung im Raume, handelt es sich um das Verbrechen(!) der Richterbestechlichkeit bzw. -bestechung nach $\$ \$ 332$ II, 334 II StGB. In $\$ 336$ StGB ist außerdem klar gestellt, dass die richterliche bzw. dienstliche Handlung ${ }^{64}$ auch in einem Unterlassen liegen kann.

$\$ 336$ StGB stellt also nicht etwa ein echtes Unterlassungsdelikt dar Mit dem dort angesprochenen »Unterlassen der Handlung « ist das Verhalten des Amtsträgers gemeint, welches zum Gegenstand der Unrechtsvereinbarung gemacht wird.

aa) Dienstausübung und Diensthandlung (\$\$331, 333 StGB) Der Begriff der »Dienstausübung « wird weit ausgelegt. Hierunter fällt jede Tätigkeit, die ein Amtsträger oder für den öffentlichen Dienst besonders Verpflichteter zur Wahrnehmung der ihm übertragenen Aufgaben entfaltet und in amtlicher Eigenschaft vornimmt ${ }^{65}$. Hierzu gehört auch und erst recht die konkrete Diensthandlung. Ausgeschlossen sind somit Privathandlungen oder Nebentätigkeiten, wie z.B. die Erteilung von Privatunterricht durch eine verbeamtete Lehrerin ${ }^{66}$. Das Vorliegen eines hinreichenden Dienstbezugs kann insbesondere bei Handlungen zweifelhaft sein, die außerhalb des eigentlichen Aufgabenbereichs des Amtsträgers liegen. Hier stellt sich die Frage, ob der Amtsträger eine Diensthandlung vornimmt oder nur gelegentlich der Dienstausübung eine außerdienstliche Handlung tätigt. Dass Geschäftsverteilungspläne und konkrete Zuständigkeitsverteilungen für die strafrechtliche Bewertung als Diensthandlung nicht entscheidend sein können, ist weit gehend anerkannt ${ }^{67}$. Andernfalls wäre z. B. der Fall nicht zu erfassen, in dem ein Amtsträger seinen faktischen Einfluss auf eine Entscheidung »verkauft « oder seine dienstliche Stellung für eine (pflichtwidrige) Handlung außerhalb des eigenen Zuständigkeitsbereichs "missbraucht». Man wird es daher als ausreichend ansehen müssen, dass die anvisierte Handlung in einem funktionalen Zusammenhang mit dem Dienstbereich des Amtsträgers steht, also eine Verbindung zu dessen allgemeinen Aufgabenkreis aufweist, und er sie bei objektiver Betrachtung »als Amtsträger« vornimmt. Zuzugeben ist aber, dass die Handhabung dieses Kriteriums im Einzelfall zu schwierigen Abgrenzungsproblemen führen $\mathrm{kann}^{68}$.

In Fall $7 a$ liegt nun ein klassischer Fall des sog. »Anfütterns«

57 Eine hiervon abweichende Regelung sah bspw. ein Entwurf des Bundesrates aus dem Jahre 1995 vor, der für die $\$ \$ 331,333$ StGB die Annahme bzw. Gewährung eines Vorteils »in Zusammenhang mit dem Amt« ausreichen lassen wollte (BR-Drs. 298/95).

58 Vgl. BGHSt 15, 239; 39, 45 (46); 49, 275 (280 ff.).

59 LG Karlsruhe NStZ 2008, 407 (408).

60 In den Handlungsalternativen »Fordern" und "Anbieten « ist nur der Wille, zu einer entsprechenden Übereinkunft über Vorteil und Gegenleistung zu kommen, festzustellen.

61 BGH NJW 2008, 3580 (3582).

62 Besonders deutlich in $\$ \$ 332,334$ StGB: »dass er eine Diensthandlung vorgenommen hat oder künftig vornehme«.

63 Zuwendungen in Hinblick auf die bloße Ausübung des Richteramtes bleiben straflos.

64 Dass die Vorschrift das Unterlassen der Dienstausübung nicht der aktiven Dienstausübung (i.S. d. $\$ \$ 331,333 \mathrm{StGB}$ ) gleichstellt ist ein - im Ergebnis unschädliches - gesetzgeberisches Versehen (vgl. SSW-StGB/ Rosenau $\$ 336$ Rdn. 3 ).

65 BGHSt 31, 264 (280); KG NJW 1998, 1877 (1878); zur teleologischen Reduktion des BGH bei Einwerbung von Wahlkampfspenden s. SATZGER JK 6/08, StGB $\$ 331$.

66 BGH GA 1966, 377.

67 BGHSt 3, 143 (145 f.); 16, 37 (38).

68 Kritik bei Bernsmann/Gatzweiler (o. Fn. 49) Rdn. 249 ff.; zum Ganzen LK/SOWADA $\$ 331 \mathrm{Rdn} .52 \mathrm{ff}$ 
vor. Bis 1997 hatte das Gesetz darauf abgestellt, dass der Vorteil in Hinblick auf eine bestimmte Diensthandlung gewährt wurde, so dass derartige Fälle nicht erfasst waren. Da hier die Gewährung eines Vorteils (Einladung zu einem Golfturnier) in Hinblick auf die allgemeine Dienstausübung des $\mathrm{M}$ erfolgt, ist der Fall nunmehr aber unter $\$ \S 331,333$ StGB zu fassen.

\section{bb) Dienstpflichtverletzung ( $\$ \$ 332,334$ StGB)}

Eine Diensthandlung ist pflichtwidrig, wenn sie gegen Gesetz, Verwaltungsvorschriften, Richtlinien, Dienstvorschriften oder einzelne Anordnungen verstöß ${ }^{69}$. Hier ist also die Handlung bzw. das Unterlassen des Amtsträgers selbständig auf seine objektive Rechtmäßigkeit hin zu überprüfen. Keinesfalls wird die dienstliche Tätigkeit allein dadurch, dass sie »erkauft" wurde, pflichtwidrig ${ }^{70}$. Des Weiteren muss die Diensthandlung - so wie sie sich nach der Unrechtsvereinbarung darstellt - auch von den Beteiligten subjektiv als Pflichtverletzung erfasst wer$\mathrm{den}^{71}$. Die Bestimmung der Pflichtwidrigkeit einer realen, in der Vergangenheit liegenden Diensthandlung hat demnach verwaltungsrechtsakzessorisch zu erfolgen und bereitet in der Regel geringe Schwierigkeiten. $\mathrm{Zu}$ denken ist an die Erteilung einer Baugenehmigung trotz nicht genehmigungsfähigen Vorhabens, Straftaten im Amt, zeitlich oder sachlich bevorzugte Bearbeitung eines Antrags oder Ermessensüber- bzw. -unterschreitungen ${ }^{72}$. Schwierigkeiten können sich ergeben, wenn ein Vorteil in Bezug auf eine künftige Diensthandlung gewährt wird. Hier legt $\$ 332$ III StGB fest, dass schon das Sich-Bereit zeigen bzgl. einer pflichtwidrigen Diensthandlung bzw. einer vorteilsbeeinflussten Ermessensausübung zur Strafbarkeit wegen Bestechlichkeit führt. Entscheidend ist somit der vom Nehmer nach außen erweckte Eindruck. Ob er sich insgeheim vorbehält, später sachgerecht zu verfahren (sog. Mentalreservation), ist unerheblich ${ }^{73}$. Geht es um eine Ermessensentscheidung ${ }^{74}$, so kommt es also nicht etwa darauf an, ob diese im Ergebnis an einem Mangel i. S. d. verwaltungsrechtlichen Ermessensfehlerlehre leidet. Vielmehr begründet allein der nach außen manifestierte Wille des Nehmers, den Vorteil in die Waagschale bei der Entscheidung zu werfen, die Strafbarkeit nach $\$ 332 \mathrm{StGB}^{75}$. Die »spiegelbildliche Regelung enthält wiederum $\$ 334$ III StGB, wo klar gestellt ist, dass auch der (erfolglose) Bestimmungsversuch die Strafbarkeit wegen (aktiver Bestechung) begründet. Für Fall $7 b$ bedeutet dies, dass sich U gem. $\$ 334$ III Nr. 2 StGB strafbar gemacht hat. Für die strafrechtliche Verantwortung des $\mathrm{M}$ bliebe zu klären, inwieweit er dem U zu verstehen gegeben hat, das Kleid (Drittvorteil!) bei der Vergabeentscheidung zu berücksichtigen. Bloßes Behalten genügt hierfür nicht ${ }^{76}$, so dass es durchaus möglich ist, dass nur U bestraft wird.

c) Gegenleistung bei \$299 StGB: Bevorzugung im Wettbewerb Bei der Bestechung im geschäftlichen Verkehr muss die Tathandlung auf eine zukünftige Bevorzugung eines anderen im Wettbewerb abzielen. Anders als bei $\$ \$ 331,333$ StGB muss die als Gegenleistung vorgesehene Bevorzugung in groben Umrissen erkennbar und festgelegt sein. Unter einer Bevorzugung wird jede Besserstellung des Täters oder eines von ihm begünstigten Dritten, auf die er oder der Dritte keinen Anspruch hat, verstanden ${ }^{77}$. Die Vorzugsbehandlung muss zudem »bei dem Bezug von Waren oder gewerblichen Leistungen « stattfinden. Die Begriffe werden (bedenklich) weit ausgelegt ${ }^{78}$. So umfasst der "Bezug « nicht nur den gesamten wirtschaftlichen Vorgang von der Bestellung über die Lieferung bis hin zur Bezahlung der Ware oder Leistung, sondern auch die sog. " umgekehrte Lieferrichtung ${ }^{79}$. Dies bedeutet, dass es dem Vorteilsgeber nicht nur um die Lieferung von Waren, sondern auch um deren Erhalt gehen kann (Bsp.: Bestechung, um ein begehrtes - weil seltenes - Gemälde zu erhalten).

In Betracht kommt hier insbesondere die vorrangige Berücksichtigung bei Aufträgen, so dass in der Variante von Fall $7 b$ eine taugliche Gegenleistung vorliegt. Die Rechtsprechung geht aber noch weiter und lässt auch die bevorzugte Bezahlung, das Aufrechterhalten bestehender Geschäftsverbindungen, das Nichtkündigen einer Geschäftsbeziehung oder den Verrat von Geschäftsgeheimnissen genügen ${ }^{80}$.

Die Bevorzugung muss zudem »im Wettbewerb« stattfinden. Dies bedeutet, dass nach Vorstellung der Beteiligten im Zeitpunkt der bevorzugenden Handlung (also nicht im Zeitpunkt der Tathandlung!) ein wirtschaftliches Konkurrenzverhältnis zwischen dem zu begünstigenden Unternehmen ${ }^{81}$ und mindestens einem anderen Unternehmen bestehen muss ${ }^{82}$.

\section{d) Regelwidrigkeit}

Das menschliche Miteinander im behördlichen wie im geschäftlichen Verkehr besteht naturgemäß aus dem gegenseitigen Nehmen und Geben von Zuwendungen. Der Beamte, der zum Dienstjubiläum eine Flasche Wein erhält, nimmt diese durchaus »für die Dienstausübung« an. Gleiches gilt für den in Fall 2 vorgestellten Staatsanwalt oder einen Beamten, der eine gesetzlich vorgesehene Gebühr erhebt. Schon diese Fälle zeigen, welch »explosiver gesetzgeberische Mix $«^{83}$ sich aus der weiten Unrechtsvereinbarung der $\$ \$ 331 \mathrm{ff}$. StGB und der Einbeziehung von Drittvorteilen ergibt. Aber auch die Anwältin, die in der Fall 5 (Variante) den Prokuristen zum Essen einlädt, denkt bei realitätsnaher Betrachtung selbst dann an eine Bevorzugung, wenn sie ihm nur einen Kaffee in ihrer Kanzlei serviert. \$299 StGB scheint also durchaus zu passen.

Die Einhaltung der Regeln der Höflichkeit oder sogar gesetzlich oder gesellschaftlich erwünschte Austauschverhältnisse zu kriminalisieren, kann aber nach allgemeiner Ansicht nicht im Sinne der Korruptionstatbestände liegen. Es setzt sich daher erfreulicherweise immer stärker die Einsicht durch, dass die Unrechtsvereinbarung nicht nur das Gegenseitigkeitsverhältnis zwischen Vorteil und Gegenleistung bezeichnet, sondern auch auf das zusätzliche Erfordernis der Sach- bzw. Regelwidrigkeit des Austauschverhältnisses verweist. Im Ergebnis geht es darum, Verhaltensweisen, die die von den Tatbeständen geschützten Rechtsgüter ${ }^{84}$ nicht ernsthaft gefährden, »normativ aus dem Bereich tatbestandsmäßigen Verhaltens [auszufiltern] $\ll^{85}$. Methodologisch kann man dies als teleologische Reduktion oder Ausschluss der objektiven Zurechnung durch Einhaltung des erlaubten Risikos begreifen.

69 BGHSt 15, 88 (92); SSW-StGB/RosenAU $\$ 332$ Rdn. 8.

70 Zuletzt BGH NStZ-RR 2008, 13 (14); weitere Nachweise bei Fischer $\S 332 \mathrm{Rdn} .7$.

71 Str.; vgl. MüKo-StGB/Korte $\$ 332$ Rdn. 24, 41; SSW-StGB/RosenaU $\S 332 \mathrm{Rdn} .13$.

72 Kasuistik bei MüKo-StGB/Konte $\$ 332$ Rdn. 9 f.; LK/Sowada $\$ 332$ Rdn. 11.

73 BGHSt 48, 44 (46)

74 Der Begriff ist in einem weiten - strafrechtlichen - Sinne zu verstehen. Erfasst sind auch der Beurteilungsspielraum bei Prüfungsentscheidungen oder der Auslegung unbestimmter Rechtsbegriffe (vgl. NK/KUHLEN $\$ 332$ Rdn. 9).

75 BGHSt 15, 239 (249); 48, 44 (46); Rengier (o. Fn. 17) $\$ 60$ Rdn. 35.

76 SSW-StGB/RosenaU $\$ 331 \mathrm{Rdn} .25 \mathrm{f}$.

77 NK/DANNECKER $\$ 299$ Rdn. 43, LACKNER/KÜHL \$299 Rdn. 5.

78 Näher (auch zu »Waren« und "gewerblichen Leistungen«) Sch/Sch/HeINE $\$ 299$ Rdn. 5 m. w. N.

79 BGHSt 2, 396 (401); 10, 269 (270); LK/Tiedemann \$299 Rdn. 31.

80 Weitere Kasuistik und Nachweise bei MüKo-StGB/DiEmer/KRICK $\$ 299$ Rdn. 16.

81 Dies wird in der Regel der Geber sein, was aber nicht zwingend der Fall sein muss.

82 S. BGHSt 49, 214 (228 f.); vgl. auch BGH NJW 2006, 3290 (3298).

83 Амвоs JZ 2003, 345 (350).

84 Dazu oben III, 2 .

85 NK/Kuhlen \$331 Rdn. 86; vgl. auch MüKo-StGB/Korte $\$ 331$ Rdn. 107 ff.; SATZGER ZStW 115 (2003), 469 (481 f.). 
aa) Regelwidrigkeit bei $\$ \$ 331 \mathrm{ff}$. StGB

Im Rahmen der Amtsträgerkorruption kann sich die Regelkonformität bzw. Sachgemäßheit des Austauschverhältnisses vor allem aus rechtlichen Erlaubnissätzen (Gesetze, Verordnungen und Satzungen, Verwaltungsvorschriften und Einzelanordnungen) ergeben ${ }^{86}$. Auf diese Weise kann dem Staatsanwalt in Fall 2 zur Straflosigkeit verholfen werden. Einen weiteren Anwendungsfall stellt die universitäre Drittmittelforschung dar. In diesem Bereich ist nach Ansicht des BGH - trotz Vorliegens einer Gegenleistung und eines Drittvorteils - der Tatbestand nicht verwirklicht, wenn das hochschulrechtlich vorgeschriebene Verfahren für die Mitteleinwerbung (Anzeige und Genehmigung) eingehalten wird ${ }^{87}$. An der fehlenden Regelwidrigkeit scheitert schließlich auch eine Bestrafung in den Fällen der Zuwendung sozialadäquater Vorteile. Hier ist nochmals auf Fall 5 zurückzukommen. In diesem Fall ist das Austauschverhältnis - amtliche Auskunft gegen Geldzahlung - deswegen regelkonform, weil niemand auf die Idee kommen würde, eine Gefährdung des Schutzgutes der $\$ \$ 331$ ff. StGB anzunehmen ${ }^{88}$. In den Fällen der Bestechung i. e. S. ( $\$ \$ 332,334 \mathrm{StGB})$ ist die Regelwidrigkeit hingegen bereits durch das Abstellen auf eine pflichtwidrige Diensthandlung belegt.

\section{bb) Regelwidrigkeit bei $\$ 299$ StGB}

Die Frage der Regelkonformität stellt sich trotz der grds. enger gefassten Unrechtsvereinbarung auch bei $\$ 299$ StGB. Gerade im Wirtschaftsverkehr ist das Streben nach Vorteilen und Bevorzugungen, nach Geben und Nehmen nicht nur weithin üblich, sondern auch im Sinne der Erhaltung und Mehrung materiellen Wohlstands unerlässlich und erwünscht. Dass auch hier die Verknüpfung (irgend)eines Vorteils mit einer Bevorzugung im Wettbewerb noch nicht für die Tatbestandsverwirklichung ausreichen soll, wird bereits daran deutlich, dass die Norm nur eine "unlautere« Bevorzugung erfassen will ${ }^{89}$. Als regelkonform einordnen können wird man wiederum sozialadäquate Vorteile sowie Drittvorteile, die dem Geschäftsherrn im Rahmen normaler Austauschverträge zufließen sollen. Obwohl in diesen Fällen der Tatbestand expressis verbis erfüllt ist, setzt sich letztlich der leistungsstärkste Anbieter durch, so dass eine Kriminalisierung aus teleologischen Gesichtspunkten ausscheiden muss. Einen Anwendungsfall einer regelkonformen Austauschbeziehung im geschäftlichen Verkehr stellt Fall 3 dar. Obwohl \$299 I StGB seinem Wortlaut nach passt, ist aus teleologischen Gründen die Tatbestandsmäßigkeit des Verhaltens des A zu verneinen.

\section{Zustimmung durch den Dienst- oder Geschäftsherren}

Die bisherigen Ausführungen haben gezeigt, dass den Korruptionstatbeständen des StGB stets eine Drei-Personen-Konstellation zu Grunde liegt: Der Geber besticht den Nehmer, der als Amtsträger oder Angestellter für einen Dritten (die öffentliche Anstellungskörperschaft oder den privaten geschäftlichen Betrieb) tätig ist. Es drängt sich daher die Frage auf, wie sich die Zustimmung des Dritten zu der Korruptionsvereinbarung auf die Strafbarkeit von Geber und Nehmer auswirkt.

\section{a) behördliche Zustimmung}

Fall 8: Der deutsche Botschafter B nimmt bei einem Festbankett in seinem Gastland einen Orden für seine »hervorragende Dienstausübung" entgegen.

Nach $\$ \$ 331$ III, 333 III StGB kann im Falle der Vorteilsannahme bzw. -gewährung (nicht aber bei der Bestechlichkeit und Bestechung!) die Genehmigung der zuständigen Behörde grundsätzlich die Strafbarkeit entfallen lassen. Zu beachten ist zunächst, dass vom Amtsträger geforderte Vorteile ${ }^{90}$ und Vorteile für richterliche Handlungen nicht genehmigungsfähig sind, da hier keine Vereinbarkeit mit der Stellung eines Amtsträgers hergestellt werden kann ${ }^{91}$. Die Genehmigung muss zudem ihrerseits rechtmäßig sein, also von der zuständigen Behörde im Rahmen ihrer Befugnisse erteilt werden ${ }^{92}$. Die vorherige Genehmigung ist in der Falllösung als Rechtfertigungsgrund zu prüfen, während die nach Vorteilsannahme erteilte Genehmigung nur einen Strafaufhebungsgrund darstellt ${ }^{93}$. Die Wirkung der nachträglichen Genehmigung als bloßer Strafaufhebungsgrund würde freilich dazu führen, dass in Fall 8 B Unrecht (!) begeht, falls er die Einholung einer vorherigen Genehmigung vergessen hätte. Da dies offensichtlich zu weit geht, wird von der h. L. auch die Möglichkeit einer mutmaßlichen Genehmigung anerkannt. Dieser Rechtfertigungsgrund greift dann ein, wenn die Einholung der vorherigen Genehmigung unmöglich oder unzumutbar war, die Zuwendung nach dem materiellen Recht genehmigungsfähig ist und der Amtsträger diesbezüglich ein subjektives Rechtfertigungselement aufweist ${ }^{94}$.

\section{b) Zustimmung des Geschäftsherren}

Fall 995: Der Vertreter einer Champagnerfirma bietet den Kellnern eines Restaurants eine Prämie dafür an, dass sie den Gästen besonders häufig jenen Champagner empfehlen. Dieses Geschäft und die Empfehlungen an die Gäste erfolgen mit Billigung des Restaurantbetreibers.

In diesem anno $1914 \mathrm{zu}$ entscheidenden Fall haben die Kellner (= Angestellte des Restaurants) sich Vorteile dafür versprechen lassen, dass sie für eine Bevorzugung der Champagnerfirma beim Bezug von Getränken sorgten. Das Reichsgericht stand nun vor der Frage, ob $\$ 299$ StGB $^{96}$ voraussetzt, dass die Unrechtsvereinbarung dem Geschäftsherren des Angestellten gegenüber verheimlicht wird. Es hat die Frage verneint und findet dafür bis heute die Unterstützung der ganz h. M. ${ }^{97}$ Zur Begründung wird in erster Linie angeführt, dass der Tatbestand dem Schutz des Allgemeininteresses an einem lauteren Wettbewerb diene, über welches der Geschäftsherr keine Dispositionsbefugnis besitze. Auch könne es für den Schutz der Mitbewerber und der Verbraucher nicht auf den Willen des Geschäftsherrn ankommen.

Dass dieses Ergebnis nicht richtig sein kann, zeigt sich bereits dann, wenn man Fall 9 nur geringfügig dahingehend umstellte, dass die Kellner die Prämien nicht selbst annehmen, sondern diese zunächst dem Restaurantbetreiber versprochen und gewährt worden wären, der sie dann an die Kellner weiterleitete. In diesem Fall läge eine straflose »Bestechung« des Geschäftsinhabers vor. Es sollte aber einleuchten, dass die vom Zufall abhängige Ausgestaltung der Vorteilsabrede - direkt an die Angestellten oder zunächst an den Geschäftsherren - nicht über die Strafbarkeit entscheiden kann ${ }^{98}$.

86 Dazu Hölt kemeier (o. Fn. 16) S. 122 f.; NK/Kuhlen $\$ 331$ Rdn. 86 ff. 87 BGHSt 47, 295 (309 ff.); krit. Höltkemeier (o. Fn. 16) S. 217 ff.; in Richtung einer Rechtfertigungslösung Mansdörfer wistra 2003, 211 (213); Korte NStZ 2003, 156.

88 Ebenso Sch/Sch/Heine $\$ 331$ Rdn. 29 a; NK/Kuhlen $\$ 331$ Rdn. 87.

89 Anders die h.M., die dem Unlauterkeitsmerkmal keine eigenständige Bedeutung einräumt (LK/TiEdEMANN $\$ 299$ Rdn. 39).

90 Auf Geberseite kommt hingegen bei allen Handlungsvarianten eine Genehmigung in Betracht (FISCHER $\$ 333$ Rdn. 11).

91 BT-Drs. 7/550 S. 271.

92 Hierzu instruktiv MüKo-StGB/Korte $\$ 331 \mathrm{Rdn}$. $153 \mathrm{ff}$.

93 SSW-StGB/Rosenau $\$ 331 \mathrm{Rdn} .50 \mathrm{ff}$.

94 Rengier (o. Fn. 17) $\$ 60$ Rdn. 42; SSW-StGB/Rosenau $\$ 331$ Rdn. 51. 95 Nach RGSt 48, 291 - Korkengeld.

96 Bzw. die Vorgängernorm $\$ 12$ UWG a.F.

97 LACKNER/KüHL $\$ 299$ Rdn. 5; FisCher $\$ 299$ Rdn. 17; LK/TiedemanN $\$ 299$ Rdn. 40.

98 So auch Rönnau StV 2009, 302 (305) m. w. N.; krit. auch SSW-StGB/ Rosenau $\$ 299$ Rdn. 25. 


\section{Auslandsfälle}

Die Globalisierung bringt es mit sich, dass sich Korruptionssachverhalte - wie auch die anfangs erwähnten Beispiele zeigen längst nicht mehr auf den innerstaatlichen Bereich beschränken. Dass im Bereich der Wirtschaftskorruption Konstellationen mit transnationalen Bezügen häufig anzutreffen sind, überrascht wenig. Jedoch könnte es theoretisch auch vorkommen, dass ausländische Polizisten deutschen Urlaubern gegenüber »die Hand aufhalten« oder deutsches Botschaftspersonal ausländischen Visabewerbern nur gegen eine "Sondergebühr« weiterhilft. Ob bei Fällen mit Auslandsbezug, deutsches Strafrecht Anwendung finden kann, ist in zwei Prüfungsschritten zu klären: Zunächst ist das Strafanwendungsrecht der $\$ \$ 3 \mathrm{ff}$. StGB durchzuprüfen, dem nachgelagert ist die Frage zu klären, ob das konkret verletzte ausländische Rechtsgut überhaupt in den Schutzbereich der deutschen Strafnorm fällt ${ }^{99}$.

\section{In- oder ausländischer Tatort}

\section{a) Inlandstaten}

Deutsches Strafrecht ist gem. $\$ \$ 3,9$ I StGB jedenfalls dann anwendbar, wenn der Tatort - also der Handlungs- oder Erfolgsort - im Inland liegt. Eine Inlandstat liegt demnach vor, soweit die Bestechungshandlung zumindest teilweise auf deutschem Territorium begangen wird, also z. B. durch Absenden eines Vorteilsangebotes, Fordern des Vorteils, Abgabe des Versprechens oder Übergabe von Bargeld ${ }^{100}$. Da sowohl $\$ \$ 331 \mathrm{ff}$. StGB als auch $\$ 299$ StGB nach h. M. abstrakte Gefährdungsdelikte darstellen $^{101}$, haben sie keinen »Erfolgsort « i. S. d. Strafanwendungsrechts ${ }^{102}$, so dass es insbesondere nicht darauf ankommt, wo die bestochene Person ihre »Gegenleistung « (also Diensthandlung/ausübung oder Bevorzugungshandlung) erbringt.

\section{b) Auslandstaten}

Soweit ein Amtsträger oder besonders Verpflichteter i. S. d. \$11 I Nrn. 2-4 StGB ${ }^{103}$ eine Bestechungstat nach $\$ \$ 331,332$ StGB im Ausland verübt, ergibt sich die Anwendbarkeit deutschen Strafrechts aus $₫ 5$ Nrn. 12, 13 StGB. Der ausländische Geber ist hingegen weder als Teilnehmer der Tat des deutschen Amtsträgers erfasst, noch kann man sagen, dass die Tat i. S. v. $\$ 5$ Nr. 14 StGB »gegen einen Amtsträger» begangen wird. Auch $\S 7$ II Nr. 2 StGB kann hier nur unter der Voraussetzung der Tatortstrafbarkeit weiterhelfen. Seit 1998 legt aber Art. $2 \$ 1$ Nr. 2 EuBestG ${ }^{104}$ fest, dass zumindest die im Ausland begangene Bestechung gegenüber einem deutschen Amtsträger ( $\$ 334 \mathrm{StGB}$ ) immer unter deutsche Strafgewalt fällt.

Für Auslandstaten nach $\$ 299$ StGB kann sich die Anwendbarkeit deutschen Strafrechts lediglich aus $\$ 7$ II StGB ergeben. Zum einen bewirkt nämlich $\$ 299$ III StGB nach fast einhelliger Ansicht keine universelle Anwendbarkeit deutschen Strafrechts nach dem Weltrechtsprinzip ${ }^{105}$. Zum anderen wird die Tat auch nicht i. S. d. \$ 7 I StGB »gegen einen Deutschen« begangen. Dies wäre nur dann der Fall, wenn die Vorschrift inländische Individualrechtsgüter schützen würde, was bei dem überindividuellen Wettbewerbsschutz gerade nicht der Fall ist ${ }^{106}$. Auch $\$ 7$ II StGB verlangt aber die Strafbarkeit am ausländischen Tatort. Hier ist grds. ausreichend, dass das Verhalten des Betroffenen unter (irgend)einem Gesichtspunkt (z. B. als Geheimnisverrat oder Untreue) strafbar ist.

\section{Schutzbereich der Tatbestände}

Die Frage, inwieweit das deutsche Korruptionsstrafrecht auch ausländischen Interessen dient, hat sich durch die Einführung von IntBestG und EuBestG (dazu sogleich) und \$299 III StGB weitgehend erledigt, kann aber für sog. Altfälle, für die gem. $\$ 2$ I, III StGB das zur Tatzeit geltende Recht gilt, noch Bedeutung erlangen. In diesem Zusammenhang gilt es festzuhalten, dass die $\$ \$ 331 \mathrm{ff}$. StGB nach einhelliger Ansicht nur die Lauterkeit der Amtsführung in Deutschland bzw. das diesbezügliche Vertrauen schützen sollten ${ }^{107}$. Dies ergibt sich schon aus der Beschränkung des Täter bzw. Adressatenkreises auf deutsche Amtsträger (vgl. $\$ 11$ I Nr. 2 StGB).

Ob $\$ 299$ StGB schon vor Einfügung von $\$ 299$ III im Jahre 2002 auch den ausländische oder zumindest europäischen Wettbewerb schützte, war Gegenstand einer Kontroverse, die sich vor kurzem an Hand des Siemens/Enel - Falles ${ }^{108}$ neu entzündete ${ }^{109}$. Der $B G H$ hat sich in seiner Revisionsentscheidung zu besagtem Fall der ganz h. M. in der Literatur angeschlossen und den ausländischen Wettbewerb für Fälle vor 2002 aus dem Schutzbereich von $\$ 299$ StGB herausgenommen ${ }^{110}$. Nunmehr stellt allerdings $\$ 299$ III StGB klar, dass korruptive Handlungen weltweit in den Schutzbereich deutschen Strafrechts fallen, also unabhängig davon, ob der Wettbewerb in Deutschland betroffen ist.

\section{Erweiterungen durch das EuBestG und das IntBestG}

Für den Bereich der Amtsträgerkorruption hat der Gesetzgeber zur Umsetzung europäischer und internationaler Rechtsinstrumente, die der Bekämpfung internationaler Korruption dienen, das Europäische und das Internationale Bestechungsgesetz - EuBest $G^{111}$ und IntBest $\mathrm{G}^{112}$ - erlassen. Beide Gesetze bewirken zum einen für Taten der Bestechung und Bestechlichkeit i.e.S. die weit gehende Gleichstellung von Amtsträgern internationaler Organisationen (insbesondere der EU) und ausländischen Amtsträgern mit ihren deutschen Kollegen. Zum anderen enthalten beide Gesetze eigene strafanwendungsrechtliche Regelungen, die den territorialen und persönlichen Geltungsbereich deutschen Strafrechts erheblich ausdehnen. Letztlich hat der externe Einfluss zu einer inhomogenen und stark verästelten Fallgruppenbildung geführt, so dass die Einzelheiten an dieser Stelle nicht erörtert werden können ${ }^{113}$.

Zumindest sei aber kurz die Frage aufgeworfen, mit welcher Legitimation sich das deutsche Strafrecht derartiger auslandsbezogener Sachverhalte annimmt. Soweit Amtsträger der EU bzw. ihrer Mitgliedstaaten betroffen sind, geht es - in Parallele zu den $\$ \$ 331 \mathrm{ff}$. StGB - um den Schutz der Lauterkeit der Verwaltungen der EU und der einzelnen Mitgliedstaaten ${ }^{114}$. Das

99 SSW-StGB/SATzger Vor $\$ \$ 3$ ff. Rdn. 7; DERs. Internationales u. Europäisches StrafR, 4. Aufl, $\$ 3$ Rdn. 9 m. w. N. auch zur Gegenansicht, die die Prüfungsfolge umkehren will.

100 Hierzu Möhrenschlager in: Dölling (Hrsg.), Handbuch der Korruptionsprävention, 8. Kap. Rdn. $402 \mathrm{ff}$.

101 Vgl. nur SSW-StGB/Rosenau $\$ 299$ Rdn. 1 bzw. $\$ 331$ Rdn. 8.

102 SSW-StGB/SAtzger $\$ 9$ Rdn. 7; Ders. (o. Fn. 100) $\$ 5$ Rdn. 25.

103 Für ausländische oder internationale Amtsträger gelten hingegen Sonderregeln; vgl. unten 3.

104 S. Fn. 112.

105 A. A. Haft/Schwoerer in FS Weber S. 367 ( $381 \mathrm{ff}$.)

106 So auch Rönnau (o. Fn. 13) III 2 Rdn. 50; anders LK/Tiedemann $\$ 299$ Rdn. 65; NK/DANNECKER $\$ 299$ Rdn. 77, die auch Individualinteressen als geschützt erachten.

107 Pars pro toto Pelz StraFo 2000, 300 m. w. N.

108 Zum ausführlichen Sachverhalt LG Darmstadt BeckRS 2007, 16611.

109 Zusammenfassend Pelz ZIS 2008, 333; SAtzger NStZ 2009, 297 (305). 110 BGHSt 52, 323 (339 ff.).

111 Gesetz zu dem Protokoll vom 27. September 1996 zum Übereinkommen über den Schutz der finanziellen Interessen der Europäischen Gemeinschaften vom 10.9. 1998 (BGBl. II S. 2340; abgedruckt bei FiscHeR Anh. 21).

112 Gesetz zu dem Übereinkommen vom 17. Dezember 1997 über die Bekämpfung der Bestechung ausländischer Amtsträger im internationalen Geschäftsverkehr vom 10.9. 1998 (BGBl. II S. 2327; abgedruckt bei FIsCHer Anh. 22).

113 Instruktiv zu den beiden Gesetzen Korte wistra 1999, 81; TINKL wistra 2006, 126.

114 MüKo-StGB/Korte $\$ 331$ Rdn. 9. 
IntBestG legt lediglich die Strafbarkeit der aktiven Bestechung internationaler und ausländischer Amtsträger im internationalen Geschäftsverkehr fest. Daher lässt sich eine gewisse Rechtfertigung aus dem Gedanken der Sicherung gleicher und fairer Wettbewerbsbedingungen im internationalen Wirtschaftsverkehr herleiten ${ }^{115}$. Vor diesem Hintergrund gibt ein noch im Gesetzgebungsverfahren befindlicher Entwurf für einen neuen $\$ 335$ a StGB Anlass zur Kritik. Die neue Vorschrift will die Bestechung und Bestechlichkeit eines internationalen oder ausländischen Amtsträgers unter Strafe stellen. Dabei soll es nicht mehr darauf ankommen, ob die Bestechungstat einen Bezug zum transnationalen Wettbewerb hat. Der Gesetzgeber meint dies mit dem Rechtsgut der Lauterkeit des öffentlichen Dienstes eines ausländischen Staates (!) bzw. des diesbezüglichen Vertrauens der Allgemeinheit rechtfertigen zu können ${ }^{116}$. Etwaigen Extremsituationen ${ }^{117}$ könne durch eine Opportunitätseinstellung nach $\S 153$ c I Nr. 1 StPO begegnet werden ${ }^{118}$. Dass diese "prozessuale Lösung« schon auf Grund ihrer kaum vorhersehbaren Anwen- dung im Einzelfall nicht befriedigen kann, sollte einleuchten, zumal völlig ungeklärt bleibt, warum das deutsche Strafrecht berufen sein sollte, jede (möglicherweise nach westlichen Maßstäben gar nicht anerkennenswerte) ausländische Verwaltung zu schützen. Der Vorwurf des "Strafrechtsimperialismus«, der schon bei Einführung des IntBestG vorgebracht wurde ${ }^{119}$, ist hier nur schwer von der Hand zu weisen.

115 MüKo-StGB/Korte $\$ 331$ Rdn. 9; TinkL wistra 2006, 126 (127); BeckOK-StGB/TrüG $\$ 331$ Rdn. 35.

116 BT-Drs. 16/6558 S. 15.

117 Bsp.: Eine deutsche Entwicklungshelferin gibt einem öffentlichen Funktionsträger in einem afrikanischen Bürgerkriegsland $100 €$, damit dieser sie unter Verletzung seiner Dienstpflichten an hungernde Flüchtlinge Lebensmittel verteilen lässt. Nach $\$ 335$ a StGB-E wären sowohl die Entwicklungshelferin als auch der ausländische Amtsträger zu bestrafen.

118 BT-Drs. 16/6558 S. 16.

119 SCHÜNEMANN GA 2003, 299 (309). 